\title{
Algorithm for detecting block-like cracks in facies of human biological fluids
}

\author{
V R Krasheninnikov ${ }^{1}$, L I Trubnikova ${ }^{2}$, O E Malenova ${ }^{1}$, A S Yashina ${ }^{3}$, \\ M L Albutova ${ }^{2}$ and $O$ A Marinova ${ }^{2}$
}

\author{
${ }^{1}$ Ulyanovsk State Technical University, 32, Severny Venetz Street, Ulyanovsk, Russia, 432027 \\ ${ }^{2}$ Ulyanovsk State University, Lev Tolstoy Street42, Ulyanovsk, Russia, 432017 \\ ${ }^{3}$ Research-and-Production Association Mars, Solnechnaya Street 20, Ulyanovsk, Russia, \\ 432022
}

\begin{abstract}
One of the most effective methods of early medical diagnosis is based on the analysis of facies (thin films) images of dried human biological fluids. The presence of special structures (markers) in facies indicates various pathologies of the organism at their earliest stages. To accelerate and reduce the cost of mass preventive medical examination of the population, it is required to create algorithms that allow markers detection on images of facies. In this paper we present an algorithm for detecting the "block-like crack" marker on images of facies of the follicular fluid obtained by puncture in vitro fertilization process. This marker indicates hypoxic and ischemic brain lesions. When developing this algorithm, first, through a visual analysis of the marker, its characteristic features were revealed. Further, methods of algorithmic detection of these features were developed. The decision about the presence of the marker was taken if there was a combination of its features. When testing this algorithm, $86 \%$ of images containing these markers were found, with $11 \%$ of false alarms.
\end{abstract}

\section{Introduction}

The development of algorithms for the automated analysis of images of the facies of biological fluids (BF) is of interest for a number of reasons. On the one hand, the processes that occur during the drying of drops of various BF (saliva, blood serum, cerebrospinal fluid, urine, etc.) are intensively studied, which can provide additional information in the diagnosis of diseases and make early diagnosis in the absence of visible symptoms, allowing treatment to begin on the early stages. On the other hand, this method belongs to the group of non-invasive methods of investigation, which is especially important in the diagnosis of diseases in newborns and premature infants. In addition, the acceleration achieved and the reduction in the cost of image analysis makes it possible to conduct mass preventive surveys of the population, helping to improve the quality of health care.

The method of studying biological fluids (BF) by means of their dehydration and analysis of the crystallization of contained substances has a long history. The theoretical description of the process of evaporation of a drop was made by D.K. Maxwell [1]. Bohlen investigated the facies of capillary blood and noticed a connection between markers and gastrointestinal tumors [2]. In the series of works by V.N. Shabalin and S.N. Shatokhina (for example, $[3,4]$ ) the features of the crystalline structures of $\mathrm{BF}$ and their connections with pathologies were analyzed. The comprehensive review and bibliography on the study of BFare given in the book by SA. Kraevoy and N.S. Coltovoy "Diagnosis by drop of blood, crystallization of biofluids" [5]. Currently, the wedge-shaped dehydration method is used to diagnose diseases in oncology, gerontology, pediatrics, obstetrics, gynecology and other fields 
of medicine. However, most of these works are aimed at identifying markers of pathologies and improving visual perception: modernization of microscopes, addition of chemical reagents in BF, etc.There are considerably fewer articles on computer processing of facies images [6-12]. And even among these works, methods of finding image zones suspicious of the existence of markers are usually developed, for example, in [10] wavelet analysis is used for this purpose. Algorithms presented in [1321] are elaborated to detect several peculiar markers with high probability while the probability of false alarms is low.

In this paper, the algorithm for detecting a marker is a block-like crack. This marker indicates the presence of structural changes in tissues, hypoxic and ischemic brain lesions.The markers on facies of the $\mathrm{BF}$ are very diverse in shape, size, orientation, etc., which is the main difficulty in their algorithmic recognition. In the present work, the following approach for the development of recognition algorithms is used. First, a visual analysis of the markers is carried out to reveal their characteristic features. Then the methods of algorithmic detection of these features are developed. The decision about the presence of the marker is made if a combination of its necessary characteristic was found in the image section.Note that only a medical worker can make the final diagnosis to the patient. Computer analysis of the facies is only auxiliary. Its destination is to identify images on which specific set of markers is present. Therefore, it is not necessary to find all markers of this type on the image. It is enough to find at least one of them and inform about it. Then the operator will perform a more thorough analysis of this image. In fact, it is required to select images which contain at least one of considered markers. This is an indicator of the effectiveness of algorithms for detecting and recognizing markers.

\section{Algorithm for block structure detection}

To develop the algorithm for detection of the marker, its main features were first revealed. They distinguish the marker from the basic structure of the facies and other markers.

\subsection{Marker description}

Most often the term "blocky" is used to denote the type of joint. The articular joint (artic trochlearis), or ginglium, is formed by a part of the block, cylinder or truncated cone on one bone and corresponding to the shape of a depression on the other [22]. It is schematically depicted in figure 1. On the left is a diagram of the block-shaped joint. On the right is its simplest schema to geometric primitives. This is more like the desired marker shape on the facies images.
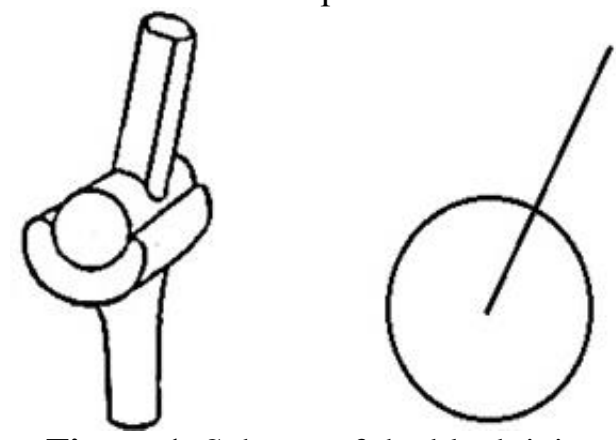

Figure 1. Scheme of the block joint.

Figures 2-6 show images of the facies of a healthy person and facies with block-like structures. Marker is recognized by a long crack and one end is enclosed in an oval. Fractions of the facies look like dark lines on the image. As can be seen from the figures, the marker has a lot of variations of form. At the end of a long crack, there may be a closed circular or oval crack or an open arcuate crack. These markers appear at the ends of radial cracks (long faults directed to the center of the facies). An exception may be some pathological conditions. With them, the line of intersection of radial cracks shifts from the center of the facies. Radial cracks are clearly visible in figure 3. 


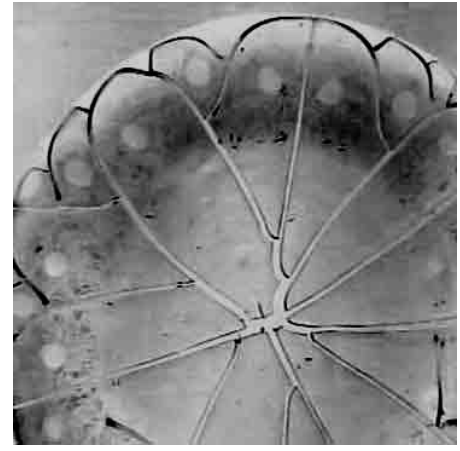

Figure 2. Bloodfacia of a healthy person.

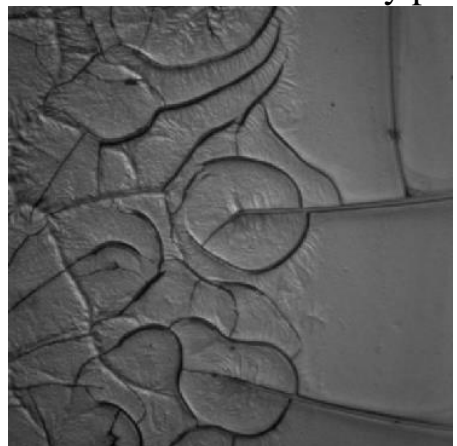

Figure 4. Block-like cracks.

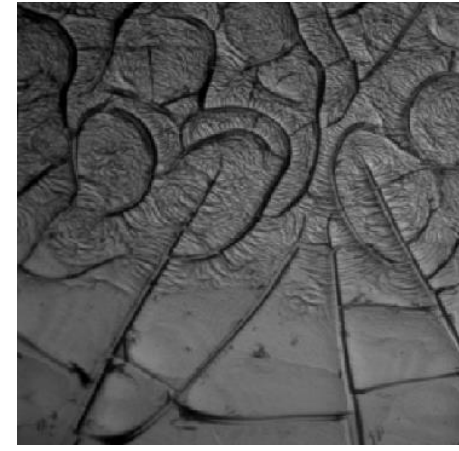

Figure 3. Block-like cracks.

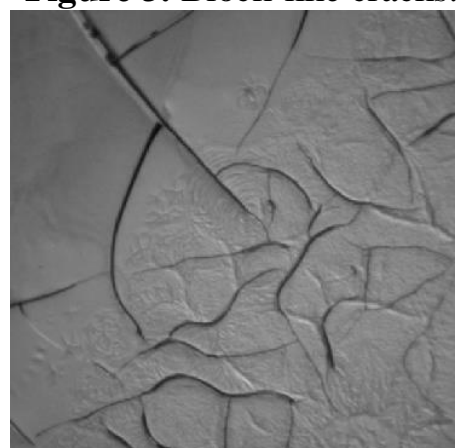

Figure 5. Block-like cracks.

\subsection{Markersearching algorithm}

Based on the characteristics described above, an algorithm for searching for a marker was compiled. It includes the following steps:

- Preparing the image for processing.

- Segment the image in brightness for highlighting cracks.

- Selection of contours.

- Search for radial cracks.

- Search for rounded cracks that intersect radial cracks.

Decision making about the presence of a marker. If the image shows the intersection of a radial crack with an arc or an ellipse, then a decision is made about the presence of a marker on the image.

\section{Description of the algorithm steps}

Let's consider the steps of the proposed algorithm operation on the example of image processing of the facies. The image shown in figure 6 is taken as example.

\subsection{Preparing the image for processing}

When you run the program, a color image is loaded into the memory. In our case, the colors of the marker do not carry any important information. Therefore, the whole image is converted to grayscale (figure 6). To reduce random noise and the number of false contours, we use a median filter.

\subsection{Brightnessimage segmentation}

Cracks are darker than the main part of the facies. For their selection, the average brightness of the image is calculated. All areas with brightness above the average are removed from further consideration (figure 7).

\subsection{Extraction of contours}

To isolate the contours, the Canny algorithm was used [23]. The result of applying it to figure 7 is shown in figure 8 . To isolate the contours boundaries by this method, the values for the two thresholds were experimentally selected. 


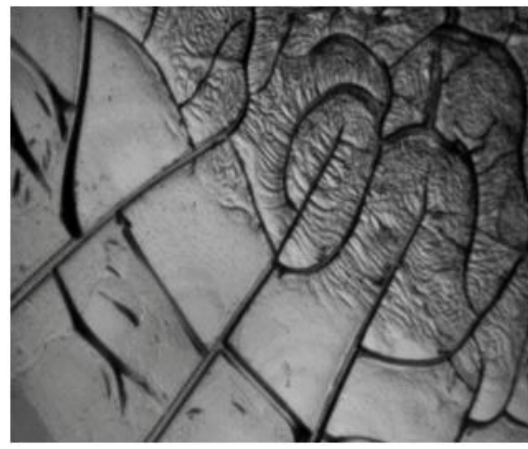

Figure 6. Uploaded image in grayscale.

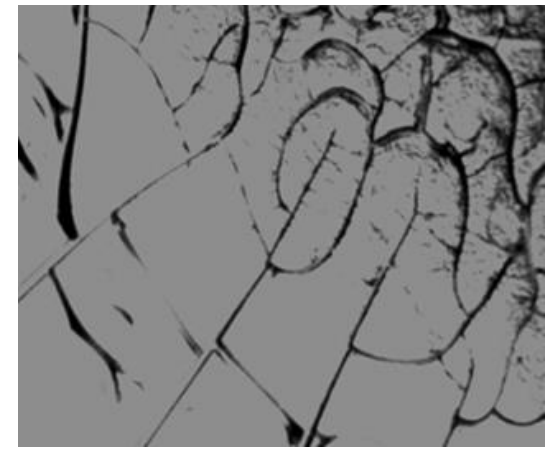

Figure 7. Threshold segmentation result.

The minimum threshold is 100 and the maximum threshold is 180 . They determine the significance of the boundary points. If the gradient value on this fragment is below the minimum threshold, then the boundary is considered to be insignificant. If there is no value on the border fragment above the maximum threshold, it is deleted. It is noticeable that there are many extraneous, useless contours (short ones). Therefore, by using per-pixel bypass of the contour, we leave only the boundaries of sufficient length. The empirically calculated threshold is 50 pixels. The bypass is implemented using the circuit bypass algorithm called "Beetle" [24]. The result is shown in figure 9.

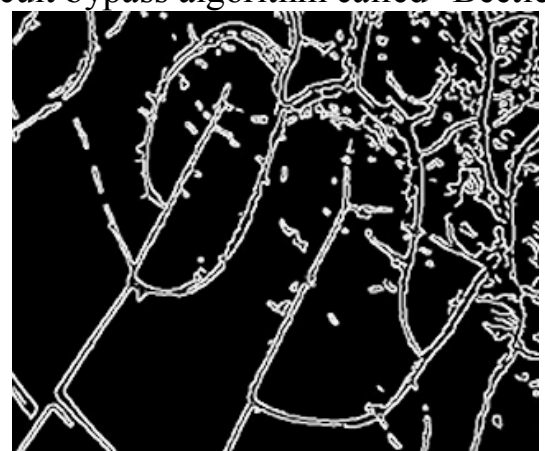

Figure 8. The result of the Canny algorithm.

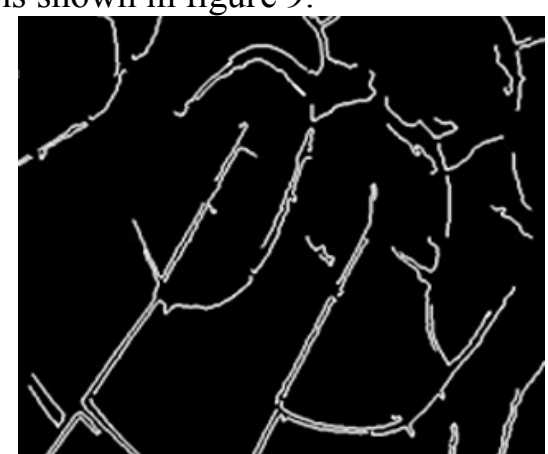

Figure 9. Insignificant contours removing.

\subsection{Search for radial cracks}

After isolating the contours, it is easy to find long approximately rectilinear cracks. For this, Huff's transformations were used [25]. With its help, long enough straight lines have been found, which can be attributed to radial cracks. It is empirically established that as $x 400$ magnification, the length of the radial crack exceeds 150 pixels. The found radial cracks are shown in figure 10.

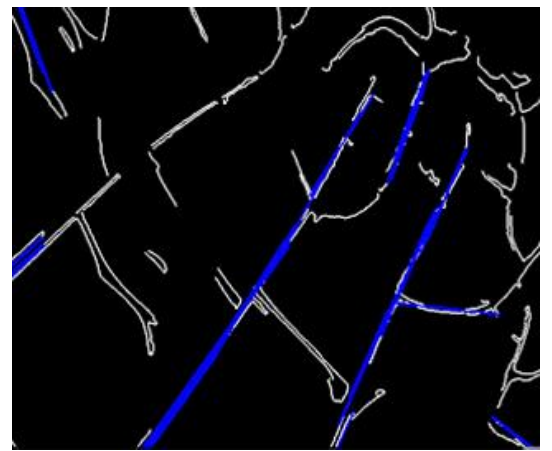

Figure 10. The result of searching for radial cracks.

\subsection{Search for rounded cracks}

The shapes of our marker vary greatly and not everyone can be an ellipse. Therefore, we use an algorithm based on the search for arcs in an image. It is described in [26] and is based on the following idea. A line similar to an arc must have the same direction of rotation (left or right) along its course. 
And the angles between consecutive segments should be in a certain confidence interval (figure 11). If the angle is too small, then the segments are considered to be collinear. They cannot be part of the arc. If the angle is too large, then the lines are part of the contour of another type (square, rectangle, etc.). In our case, the confidence interval of the angle of rotation was chosen experimentally from 6 to 60 degrees. Since the bypass goes in a specific direction, parts of the arc will be considered as vectors (figure 12). The angle between them is determined by the relation:

$$
\theta_{i}=\arccos \left(\frac{\overline{v_{i+1}} \overline{v_{i}}}{\left|\overline{v_{i+1}}\right|\left|\overline{v_{i}}\right|}\right) .
$$

The detected arcs are shown in figure 13.

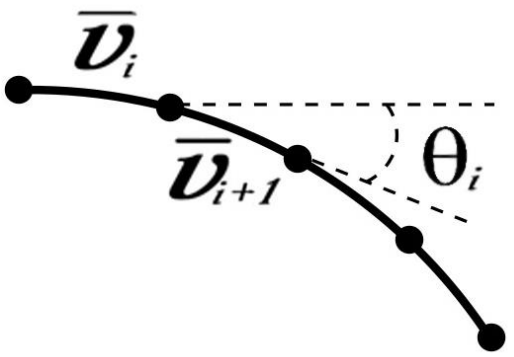

Figure 11. Arrangement of arc segments.

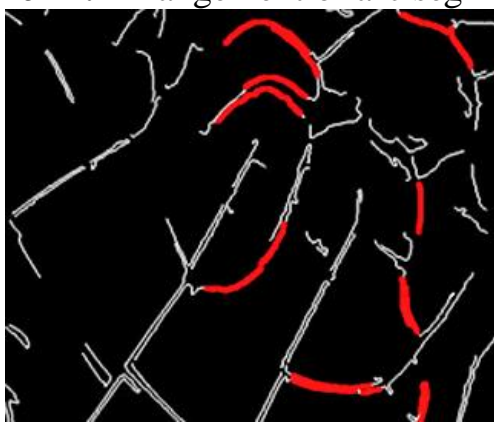

Figure 13. The found arcs.

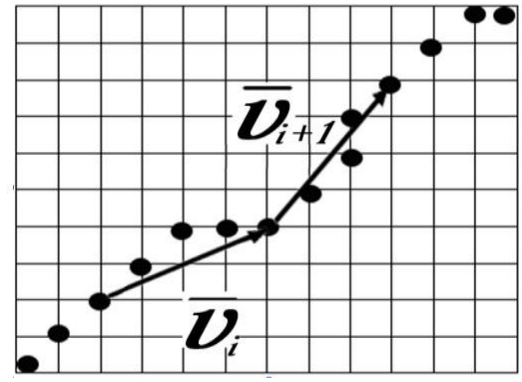

Figure 12. Vectors on the line.

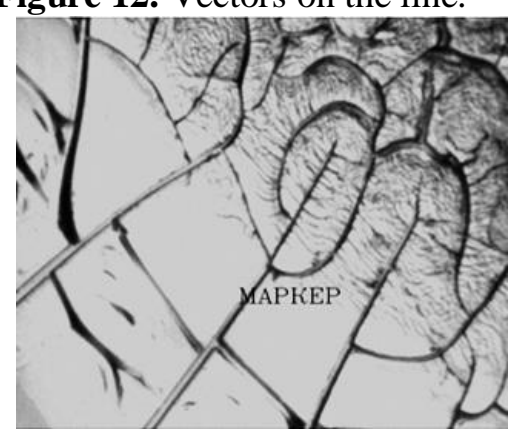

Figure 14. Discovered marker.

\subsection{Decision-making}

We check the angles of intersection of the found arcs and radial cracks. If the angle found is close to a direct angle, then a decision is made about the presence of a block-like crack in the facies image. In figure 14, the inscription indicates the found marker.

\section{Experimental results}

The purpose of image processing facies is to identify images on which there are desired markers (in our case - block-like cracks). In this case, it is not necessary to find all markers of this kind, it suffices to indicate that such markers (at least one) are available. Therefore, when testing the described algorithm, it was considered that the detection occurred if at least one block-like crack was found on the image on which they were available.

The algorithm was tested on 300 images of blood serum facies of patients examined at the medical faculty of Ulyanovsk State University. As a result of the tests, $86 \%$ of images with the desired markers were correctly identified. To find the probability of false alarms, this algorithm processed image facies, on which this marker is absent. It turned out that on $11 \%$ of such images false detections of structures were made, somewhat similar to the desired marker. This suggests that in the future it is advisable to extend the set of features of this marker for more accurate identification.

\section{Conclusion}

As a result of this study, an algorithm and software were developed for the automated detection of facies images with suspicion of the presence of a "block-like crack" marker and the screening of 
images without this marker. Tests showed that $86 \%$ of the images on which markers were present were properly identified. False detections of structures were made on $11 \%$ of images without this marker. But it found somewhat similar to the desired marker. This indicates that in the future it is expedient to extend the set of characteristics of this marker for more accurate identification. It should be noted that among the images from the list of false alarms there were other markers, which are some kind of "suspicious areas". Their search is also included in the tasks of detection algorithms for markers.

\section{References}

[1] Maxwell J C 1890 The Scientific Papers of James Clerk (Niven University Press Cambridge) p 211

[2] Bolen H L 1942 The Blood Pattern as a Clue to the Diagnosis of Malignant Disease J Lab Clin Med 27 1522-1536

[3] Shatokhina S N and Shabalin V N 2013 Atlas of structures of human noncellular tissues in norm and pathology: Morphological structures of blood serum (Moscow-Tver: Triad) p 240

[4] Shatokhina S N and Shabalin V N 2001 Morphology of human biological fluids (Moscow: Chrysostom) p 304

[5] Kraevoy S A and Koltovoy N A 2016 Diagnosis using a single drop of blood. Biofluid crystallization (Moscow-Smolensk)

[6] Abdumanonov A A, Botirov M T and Karabaev M K 2014 Development of computer programs and algorithms for automatic morphometry bioliquid facies New look. International Scientific Gazette 6

[7] Buzoverya M E, Shishpor I V, Sevastyanov V I and Perov N V 2011 The hardware and software complex "Morfo" (Sarov: RFJC-VNIIEF) 254-255

[8] Ilyasova N Yu, Kupriyanov A V and Khramov A G 2012 Information technologies of image analysis in problems of medical diagnostics (Moskow : Radio and communication) p 424

[9] Lukashevich M M 2013 Analysis of images of biological fluids Bulletin of the Fund for Fundamental Research 3 94-105

[10] Makarevich V E, Kirilenko E A, Petrashenko V A, Zablotskaya T U and Bilokon T A 2014 Methods of wedge dehydration of biological fluids Morphology 8(1) 113-117

[11] Petrov V O 2009 Automation of the analysis of raster images of a solid phase of a biological fluid of biomedical preparations (Volgograd: VSTU) $150 \mathrm{p}$

[12] Pogodin S V 2010 Automatic diagnosis on the analysis of medical images Collection of scientific works of the 1st International teleconference "Problems and perspectives of modern medicinebiology and ecology 171

[13] Krasheninnikov V R and Kopylova A S 2011 Identification of Pectinate Structures in Images of Blood Serum Facia Pattern Recognition and Image Analysis 21(3) 508-510

[14] Krasheninnikov V R and Kopylova A S 2012 Algorithms for automated processing images blood serum facies Pattern Recognition and Image Analysis 22(4) 583-592

[15] Krasheninnikov V R, Trubnikova L I, Albutova M L, Kopylova A S and Tarasova A V 2013 Algorithm for detecting language structures on images of blood serum Ulyanovsk medicobiological journal 4 70-73

[16] Krasheninnikov V R, Trubnikova L I, Albutova M L, Kopylova A S and Tarasova A V 2013 Detection of sickle-shaped cracks in images of facies of cervical mucus of a woman Ulyanovsk medico-biological journal 4 81-85

[17] KrasheninnikovV R,Trubnikova L I, Albutova M L and Yashina A S 2015 Algorithm for detecting a marker of gallbladder disease on images of blood serum Ulyanovsk Medical and Biological Journal 4 104-110

[18] Krasheninnikov V R, Yashina A S and Malenova O E 2017 Markers detection on facies of human biological fluids Procedia Engineering 201 312-321

[19] Krasheninnikov V R, Yashina A S and Malenova O E 2017 Algorithms for detection of markers on the facies of human biological fluids Proceedings of the III International Conference and Youth School "Information Technologies and Nanotechnologies" (ITNT-2017) 655-662 
[20] Krasheninnikov V R, Yashina A S and Malenova O E 2017 Algorithms of crescent structure detection in human biological fluid facies The International Archives of the Photogrammetry Remote Sensing and Spatial Information Sciences 4 169-172

[21] Zakharova G P and Shabalin V V 2014 Computer identification of distinctive features and anomalies in samples of multicomponent solutions of biological fluids Russian Otorhinolaryngology 6 37-42

[22] Vorobiev V P 1940 Atlas of human anatomy (Leningrad: Medgiz) p 382

[23] Canny J A 1986 Computational Approach to Edge Detection IEEE Transactions on Pattern Analysis and Machine Intelligence 6 679-698

[24] Potapov N N and Tupikov P A 2007 Methods for selecting contours in images 8th international conference Image recognition and image analysis: new information technologies 105-108

[25] Ballard D H 1981 Generalizing the Hough Transform to direct arbitrary shapes Pattern Recognition 13 111-122

[26] Akinlar C and Topal C 2013 ED Circles: A real-time circle detector with a false detection control Pattern Recognition 46 725-740 